\title{
The Influence of Laser Pulse Duration on Periodic Surface Structures on Stainless Steel
}

\author{
Paulina Dzienny ${ }^{*}$, Bogusz Stępak ${ }^{*}$, Aleksander Budnicki* $^{* *}$ and Arkadiusz Antończak ${ }^{*}$ \\ ${ }^{*}$ Faculty of Electronics, Wroclaw University of Science and Technology, \\ Wyb. Wyspiańskiego 27, Wrocław 50-370, Poland \\ E-mail: paulina.dzienny@pwr.edu.pl \\ ** TRUMPF Laser GmbH + Co. KG, Aichhalder Straße 39, Schramberg D-78713, Germany
}

\begin{abstract}
Laser-induced periodic surface structures (LIPSS) are highly periodic wavy surface features which can be generated with deposition of a polarized radiation on almost any material. Usually, research focuses mainly on structures obtained with one specified pulse duration. In this study LIPSS formed on electropolished stainless steel under irradiation of nano-, pico- and femto-second pulse duration time were compared. The surface morphology of samples was determined by atomic force microscopy and optical microscopy. The chemical composition of surfaces was examined using energy-dispersive X-ray spectroscopy.
\end{abstract}

DOI: $10.2961 /$ jlmn.2020.01.2003

Keywords: LIPSS, ripple, nanostructures, laser micromachining

\section{Introduction}

Laser-induced periodic surface structures (LIPSS), known also as ripples [1], periodic patterns or selforganizing nanostructures [2], are studied for more than 50 years, since they were first observed by Birnbaum in 1965 [3]. They can be obtained on virtually any material, including metals [4], semiconductors [5] and dielectrics [6] upon irradiation with polarized radiation. These structures can be generated in single step process, that enables surface functionalization leading to control of mechanical, optical and even chemical properties of the surface. The periods of surface ripples induced by long-pulse lasers are usually close to the laser wavelength $\lambda$, whereas short and ultrashort pulse laser give the possibility of obtaining structures with periods significantly smaller than $\lambda$. Their orientation depends on the laser light polarization. Several explanations for LIPSS formation phenomenon were proposed [7]. The potential for their application is wide, among others in biomedical field [8], decorative [9], or mechanical [10]. A particularly interesting application is the use of nanoripple patterns as protection against counterfeiting [4].

The motivation of this research was to gain information about the morphology of LIPSS structures obtained on stainless steel with radiation of varying pulse duration. Usually, research focuses on structures obtained with a specified one [11]. In dependence of pulse duration dynamics of the formation process changes, what leads, consequently, to significantly distinct surface morphology. Comparison of such structures may give us important information about which process parameters are optimal for creating structures of best properties for specified application.

\section{Material and experiment}

As a material electropolished stainless steel AISI 304 from Valley Design Corp. was used. Each piece was square with a side of $25 \mathrm{~mm}$ and $1 \mathrm{~mm}$ thick. The surface roughness was $<20$ angstroms. In the experiment two laser sys- tem were used: Nd:YAG DS Series laser from Photonics Industries International (PII), Inc. (as nanosecond laser source) and TRUMPF TruMicro 2020 fiber laser (tunable form 300 fs to $20 \mathrm{ps}$ ) - Table 1.

Table 1 Laser system parameters

\begin{tabular}{|c|c|c|}
\hline Laser system & TruMicro 2020 & PII Laser \\
\hline Wavelength $[\mathrm{nm}]$ & 1030 & 1064 \\
Pulse duration time & $300 \mathrm{fs}-20 \mathrm{ps}$ & $24 \mathrm{~ns}$ \\
Pulse repetition rate $[\mathrm{kHz}]$ & 40 & 40 \\
Spot diameter $[\mu \mathrm{m}]$ & 100 & 40 \\
Scanning speed $[\mathrm{mm} / \mathrm{s}]$ & 200 & 200 \\
\hline
\end{tabular}

The matrices of irradiated samples were designed to cover wide range of both power (laser fluence) and overlapping (figure 1). Each processed field size was $0.5 \times 0.5$ $\mathrm{mm}^{2}$. The samples were irradiated with raster mode using focused linearly polarized laser beam and galvanometric scanner. The laser beams were focused on the target through a $163.2 \mathrm{~mm}$ focal length f-Theta Lens. The diameter of the beam at focal plane was about $100 \mu \mathrm{m}$ for fs/ps laser and about $40 \mu \mathrm{m}$ for ns laser. In all cases shown in this article the distance between scan lines was $5 \mu \mathrm{m}$. The pulse repetition rate was $40 \mathrm{kHz}$ and scanning speed 200 $\mathrm{mm} / \mathrm{s}$. All experiments were conducted in air environment. The surface morphology of samples was determined by atomic force microscopy (AFM) using Nanosurf FLEXAxiom in contact mode, scanning electron microscopy (SEM) using SEM/Xe-PFIB FEI Helios PFIB microscope and optical digital microscopy - KEYENCE VHX 5000. The basic chemical analysis was carried out using energydispersive X-ray spectroscopy (EDS) technique. The ablation threshold for ns pulse duration was $2.1 \mathrm{~J} / \mathrm{cm}^{2}$, for $\mathrm{ps}$ $4.8 \mathrm{~mJ} / \mathrm{cm}^{2}$ and for fs $3.8 \mathrm{~mJ} / \mathrm{cm}^{2}$. 


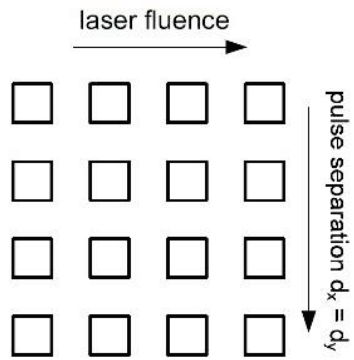

Fig. 1 Test matrix scheme. Each processed field size was $0.5 \times 0.5 \mathrm{~mm}^{2}$. Laser fluence varied from 10.8 to $62.4 \mathrm{~mJ} / \mathrm{cm}^{2}$ with $0.63 \mathrm{~mJ} / \mathrm{cm}^{2}$ steps for fs and ps pulse duration, and from 1 to $4.8 \mathrm{~J} / \mathrm{cm}^{2}$ with $2.2 \mathrm{~mJ} / \mathrm{cm}^{2}$ steps for ns and step of translation from 3 to $7 \mu \mathrm{m}$.

\section{Results and discussion}

The analysis was performed for selected squares from test matrices - one for each duration time (table 2). They were chosen based on preliminary microscopic observations as the most regular and of best quality. The periodicity of the ripples was estimated based on fast Fourier transform (FFT) of optical microscopy images (table 2d). The analyzed microstructures form a continuous onedimensional diffraction grating with a period of about $1 \mu \mathrm{m}$, which corresponds to the laser wavelength, hence low spatial frequency LIPSS (LSFL) were obtained.

Table 2 AFM images of LIPSS samples prepared with laser irradiation of $300 \mathrm{fs}, 20 \mathrm{ps}$ and $24 \mathrm{~ns}$ pulse duration time

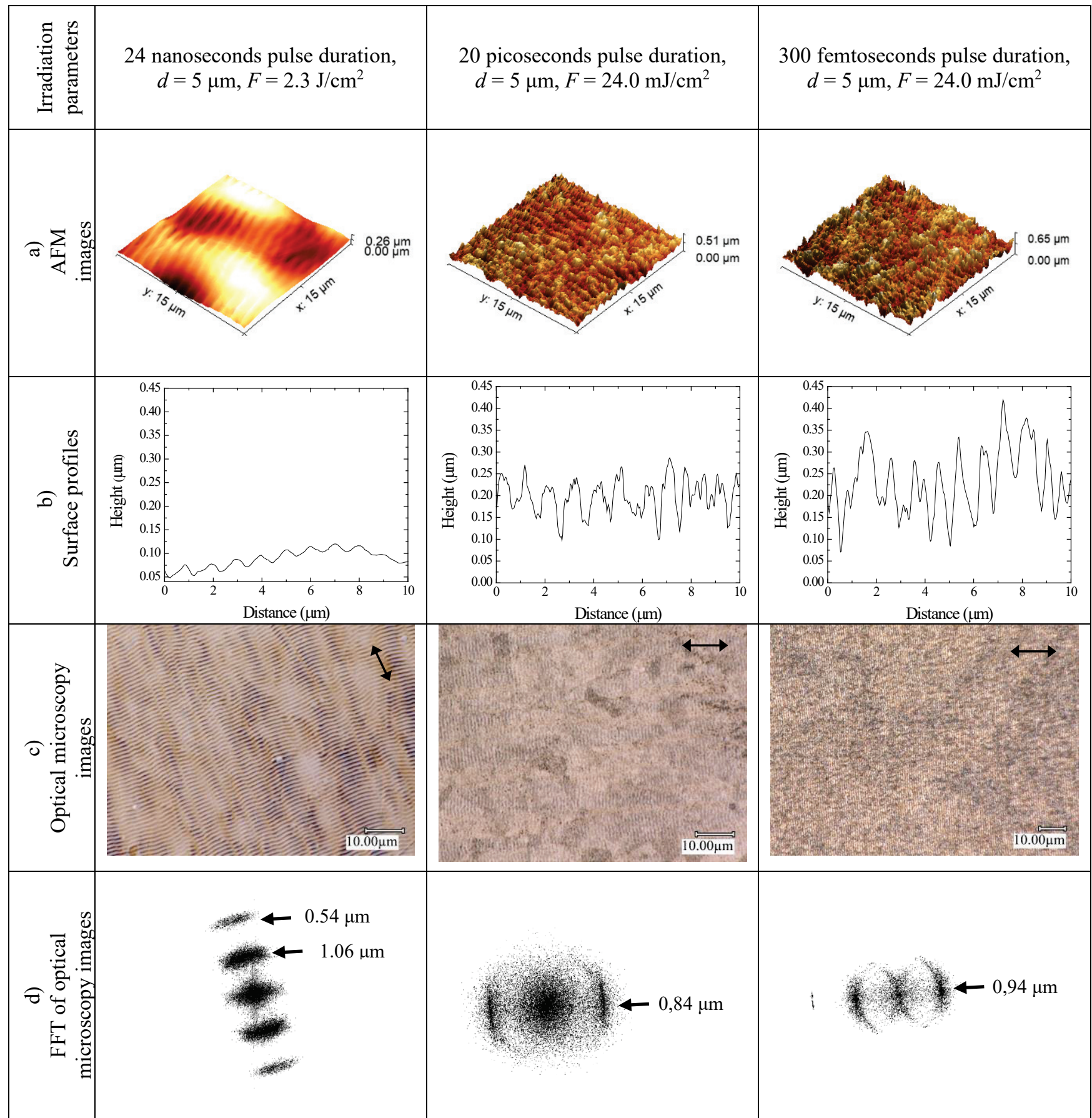


The periodicity of around $0.5 \mu \mathrm{m}$ in FFT for nanosecond pulse duration is due to clear distinction on microscopic image between ripples and intervals - the algorithm classified them as another pattern. For LIPSS obtained under pico- and femtosecond pulse duration irradiation periodicity decreases with the increase of deposited energy. Also, as it is shown in table 2d, FFT gives information about the quality of the structures: periodicities present and dispersion of structures direction. LIPSS orientation was the same for all prepared samples.

Optical microscopy images showed that in the case of samples prepared with nanosecond pulse surface is probably covered with oxides. Profile of samples obtained by AFM analysis show that nanoripples obtained with ns are shallow and smooth in comparison with pico- and femtosecond (table $2 b$ ). The main reason for this phenomenon can be both melting and surface oxidation in case of ns irradiation - a significant amount of energy is transferred to heat and transported deep into the material. This in turn enables heat accumulation. In the case of pico- and femtosecond lasers, thermal processes also occur, which has been demonstrated several times in the literature [12][13][14], but the melting of the material does not occur during pulse deposition because of the lack of equilibrium between electrons and lattice. Thus, smoothing of ripples due to molten material transport is limited in this case as far as we assume that ripples are being formed due to SPP effect during the energy deposition. Otherwise, during nanosecond pulse deposition material is simultaneously being melted what allows ripple smoothening. Also, ionized material on the surface has longer time to react with the electromagnetic field.

To assess the change in the chemical composition of the surface, the EDS analysis on chosen samples was performed. It shows no changes in chemical composition of surfaces treated with radiation of pico- and femtosecond pulse duration, whereas in nanosecond case there is an increase in oxygen content. The oxidation of surface is the result of sample heating during the process.

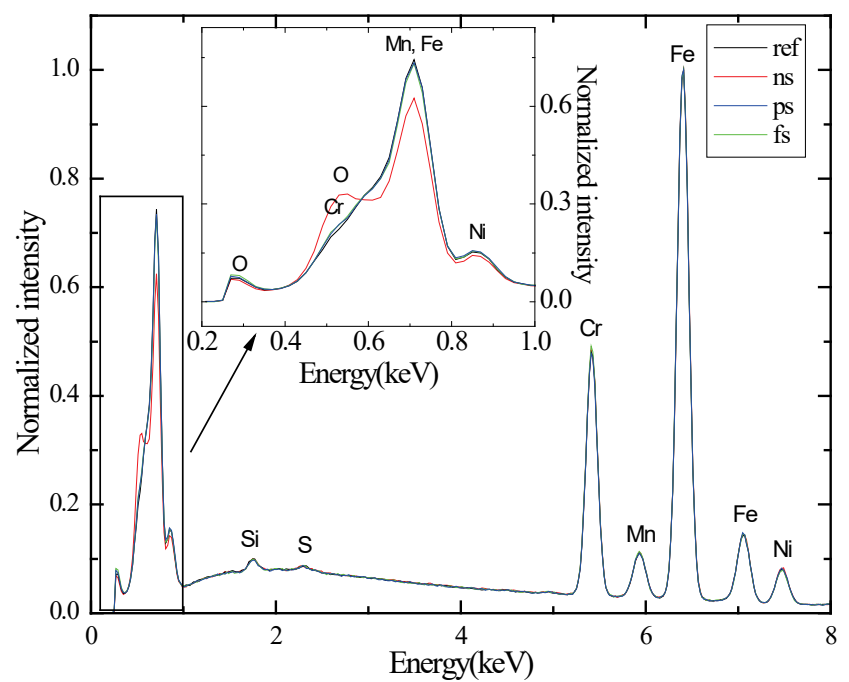

Fig. 2 EDS analysis Fig. 4. EDS analysis of LIPSS samples prepared with laser irradiation of $300 \mathrm{fs}, 20 \mathrm{ps}$ and $24 \mathrm{~ns}$ pulse duration time.
However the change seems to be insignificant and should be regarded as indicative - it should be noticed that EDS technique penetrates into the sample much deeper than the thickness of modified layer. The acceleration voltage was $15 \mathrm{kV}$, so it can be assumed that the information depth is around $1 \mu \mathrm{m}$. This result suggests that on the sample surface mainly chromium oxide is created $\mathrm{Cr}_{2} \mathrm{O}_{3}$, since it has the most negative free energy of formation of all the likely oxidation products [15]. Another possibility is the formation of a duplex oxide layer - an outer iron layer oxide phase with an inner mixed iron-chromium layer that probably can be a spinel of the type $\mathrm{Fe}(\mathrm{Fe}, \mathrm{Cr})_{2} \mathrm{O}_{4}$ [16]. It is to be investigated. On samples obtained with femto- and picosecond pulse duration nanoripple degrade with an increase of pulse energy (table 3a). First, discontinuous LIPSS can be observed for laser fluence $10 \mathrm{~mJ} / \mathrm{cm}^{2}$. In case of nanosecond radiation first structures appear for laser fluence 1 $\mathrm{J} / \mathrm{cm}^{2}$ (table 3b). With the increase of power surface is covered in thick layer of oxides and for fluence above $3.9 \mathrm{~J} / \mathrm{cm}^{2}$ LIPSS do not occur. Interesting side-effect was obtained for samples irradiated with picosecond pulses the crystallographic structure of material was revealed (fig. 3). It is due to that onset of LIPSS formation is crystalorientation dependent [17]. Effect was observed for samples prepared with pulse duration time from 5 to $20 \mathrm{ps}$, which is the maximum value for used laser system.

a)

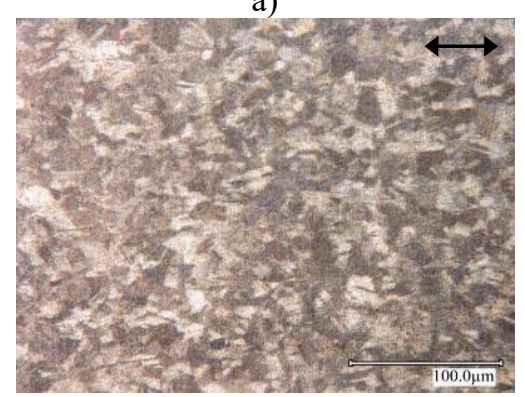

b)

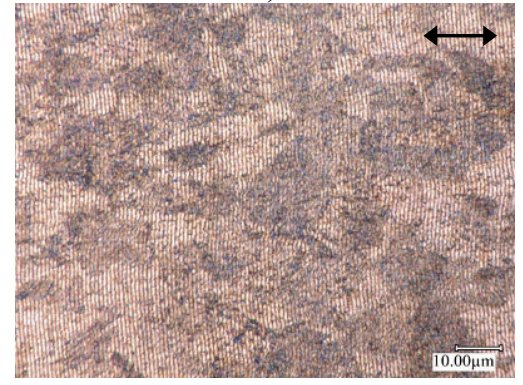

c)

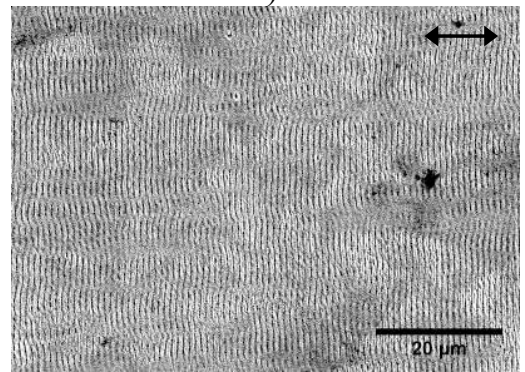

Fig. 3 a) and b) optical microscopy images of varying magnification c) and SEM image of sample prepared with laser irradiation of $20 \mathrm{ps}$ pulse duration and $31 \mathrm{~mJ} / \mathrm{cm} 2$ with visible crystallographic structure. 
Table 3 Microscopic images of LIPSS samples prepared with laser irradiation of a) $300 \mathrm{fs}$ and b) 24 ns pulse duration time - degradation with power increase; for all cases $d=5 \mu \mathrm{m}$

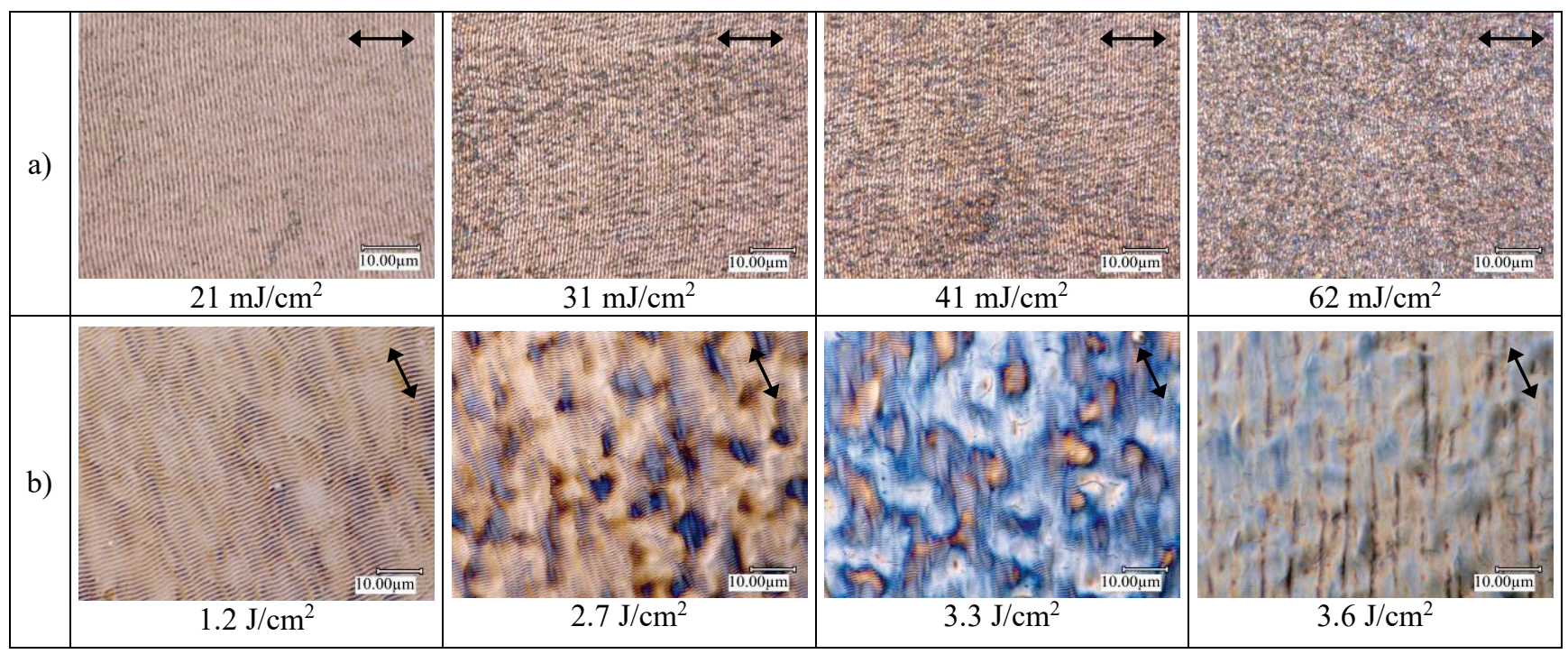

\section{Summary}

The formation of laser induced periodic surface structures under irradiation of varying pulse duration in air environment was analyzed. The process parameters suitable for homogenous structuring were defined. The samples were examined by EDS. The surfaces irradiated with nanosecond pulse have higher concentration of oxygen in comparison with reference surface and other samples which is in line with expectation. Optical microscopy images showed that in the case of samples prepared with nanosecond pulse surface is covered with oxides. Profile of samples obtained by AFM analysis show that nanoripples obtained with ns are shallow and smooth in comparison with pico- and femtosecond. For samples irradiated with picosecond pulse interesting effect occurred - the crystallographic structure of material was revealed. This method can be a potential promising alternative to chemical etching for crystallographic analyses. It is to be investigated.

\section{Acknowledgement}

This research was conducted in cooperation with TRUMPF Laser, Germany and was supported by Department of Field Theory, Electronic Systems and Optoelectronics (statutory funds no. 0401/0031/2018), Wroclaw University of Science and Technology and by the Polish National Agency for Academic Exchange (contract no. PPI/APM/2018/1/00031/U/001).

\section{References}

[1] J. V. Obona, V. Ocelik, B. Visser, J. Th. M. De Hosson, J. Z. P. Skolski, V. S. Mitko, G. R. B. E. Romer, A. J. Huis in't Veld: Proc. 24th Int. Conf. on Surf. Modif. Technol. (SMT 24), 31, (2010) 1.

[2] G. R. B. E. Römer, A. J. Huis in't Veld, J. Meijer, and M. N. W. Groenendijk: CIRP Ann. - Manuf. Technol.,
$58,(2009) 201$.

[3] M. Birnbaum: J. Appl. Phys., 36, (1965) 3688.

[4] E. I. Ageev, V. P. Veiko, E. A. Vlasova, Y. Y. Karlagina, A. Krivonosov, M. K. Moskvin, G. V Odintsova, V. E. Pshenichnov, V. V. Romanov and R. M. Yatsuk: Opt. Express, 26, (2018) 2117.

[5] J. Bonse, A. Rosenfeld, and J. Krüger: J. Appl. Phys., 106, (2090) 104910.

[6] D. Dufft, A. Rosenfeld, S. K. Das, R. Grunwald, and J. Bonse: J. Appl. Phys., 105, (2009) 034908.

[7] J. Bonse, S. Höhm, S. V. Kirner, A. Rosenfeld, and J. Krüger: IEEE J. Sel. Top. Quantum Electron., 23, (2017) 9000615.

[8] E. Rebollar, S. Pérez, M. Hernández, C. Domingo, M. Martín, T. Ezquerra, J. P. García-Ruiz and M. Castillejo: Phys. Chem. Chem. Phys., 16, (2014) 17551.

[9] J. Yao, C. Zhang, H. Liu, Q. Dai, L. Wu, S. Lan, A. V. Gopal, V. A. Trofimov and T. M. Lysak: Appl. Surf. Sci., 258, (2012) 7625.

[10]J. Eichstät, G. R. B. E. Römer, and A. J. Huis in’t Veld: Phys. Procedia, 12, (2011) 7.

[11]J. G. A. B. Simões, R. Riva, and W. Miyakawa: Surf. Coatings Technol., vol. 344, (2018) 423.

[12]N. Singh: Int. J. Mod. Phys. B, 24, (2010) 1141-1158.

[13]Z. Lin, L. V. Zhigilei, and V. Celli: Phys. Rev. B Condens. Matter Mater. Phys., 77, (2008) 1.

[14]F. Bauer, A. Michalowski, T. Kiedrowski, and S. Nolte: Opt. Express, 23, (2015) 1035.

[15]K. M. Łęcka, A. J. Antończak, B. Szubzda, M. R. Wójcik, B. D. Stępak, P. Szymczyk, M. Trzciński, M. Ozimek and K. M. Abramski: J. Laser Appl., 28, (2016) 032009.

[16]G. C. Allen, J. M. Dyke, S. J. Harris, and A. Morrist: Oxid. Met., 29, (1998) 391.

[17]X. Sedao, C. Maurice, F. Garrelie, J.-P. Colombier, S. Reynaud, R. Quey and F. Pigeon: Appl. Phys. Lett., 104, (2014) 1.

(Received: June 17, 2019, Accepted: January 20, 2020) 